OPEN ACCESS

Edited by:

Hua Tan,

University of Texas Health Science

Center at Houston, United States

Reviewed by:

Moira Garraus,

Hospital Sant Joan de Déu Barcelona,

Spain

Zhimin Liu,

Janssen Pharmaceuticals, Inc.

United States

*Correspondence:

Zheniian Zhuo

zhenjianzhuo@163.com

Specialty section:

This article was submitted to

Pediatric Oncology,

a section of the journal

Frontiers in Oncology

Received: 03 April 2021

Accepted: 05 July 2021

Published: 21 July 2021

Citation

Chen H, Guan Q, Guo H, Miao L and Zhuo Z (2021) The Genetic Changes of Hepatoblastoma.

Front. Oncol. 11:690641. doi: 10.3389/fonc.2021.690641

\section{The Genetic Changes of Hepatoblastoma}

\author{
Huitong Chen ${ }^{1}$, Qian Guan ${ }^{1,2}$, Huiqin Guo ${ }^{1,2}$, Lei Miao ${ }^{1}$ and Zhenjian Zhuo ${ }^{1 *}$ \\ 1 Department of Pediatric Surgery, Guangzhou Institute of Pediatrics, Guangdong Provincial Key Laboratory of Research in \\ Structural Birth Defect Disease, Guangzhou Women and Children's Medical Center, Guangzhou Medical University, \\ Guangzhou, China, 2 School of Medicine, South China University of Technology, Guangzhou, China
}

Hepatoblastoma is the most common malignant liver cancer in childhood. The etiology of hepatoblastoma remains obscure. Hepatoblastoma is closely related to genetic syndromes, hinting that hepatoblastoma is a genetic predisposition disease. However, no precise exposures or genetic events are reported to hepatoblastoma occurrence. During the past decade, significant advances have been made in the understanding of etiology leading to hepatoblastoma, and several important genetic events that appear to be important for the development and progression of this tumor have been identified. Advances in our understanding of the genetic changes that underlie hepatoblastoma may translate into better patient outcomes. Single nucleotide polymorphisms (SNPs) have been generally applied in the research of etiology's exploration, disease treatment, and prognosis assessment. Here, we reviewed and discussed the molecular epidemiology, especially SNPs progresses in hepatoblastoma, to provide references for future studies and promote the study of hepatoblastoma's etiology.

Keywords: hepatoblastoma, etiology, genetics, single nucleotide polymorphism, epidemiology

\section{INTRODUCTION}

Hepatoblastoma arising from the hepatocyte precursor is the most common malignant liver tumor among children (1). The typical clinical symptoms of hepatoblastoma are alpha-fetoprotein (AFP) rising and abdominal mass (2). Due to the rarity of hepatoblastoma, diagnosis and treatment are facing challenges. With medical-technical development such as adjuvant chemotherapy and hepatectomy in decades, the 5 -year survival rate is greater than $70 \%$ nowadays $(3,4)$. Despite the improved survival rate, numerous survivors suffer treatment-related side effects, such as hearing loss or cardiomyopathy (5). In addition, the prognosis of advanced stage hepatoblastoma patients with unresectable tumors remains poor (6).

Unlike the hepatocellular carcinoma which has clear pathogenesis (HCC), the etiology of hepatoblastoma has no connection with hepatitis B virus or cirrhosis (7). The first study of geneticmolecular changes in hepatoblastoma was conducted in the late 1980s (8). However, the etiology of hepatoblastoma remains unclear by far. In this review, we aimed at giving a brief overview of the molecular epidemiology for hepatoblastoma, focusing on the SNPs that influence hepatoblastoma risk. We further discussed the clinical challenges for elucidating the etiology of hepatoblastoma and provided theoretical basis for future prevention, diagnosis, and therapeutic approaches for hepatoblastoma. 


\section{EPIDEMIOLOGY}

Because of the rarity of hepatoblastoma, the hepatoblastoma's epidemiology has not been investigated comprehensively. Majority of hepatoblastoma are sporadic and are commonly found in children in their first 5 years, with a predominance in boys (9). The incidence of hepatoblastoma remains at a lower level worldwide comparing with other solid tumors in children, including neuroblastoma and Wilms tumor (10-12). Employing the Surveillance, Epidemiology and End Results (SEER) database, the incidence of hepatoblastoma in the United States was $1.5-1.9$ per million with an upward tendency $(5,13)$. It is similar to the incidence rate of roughly 1.1 per million in China and 1.7 per million in the Nordic countries $(14,15)$. There is little difference of hepatoblastoma's incidence in diverse countries, but these data were not collected from the same period. The difference in ethnicity is also correlated to the incidence rate. It was reported that the incidence rate in blacks was relative lower (16). A population-based analysis conducted in the United States revealed that the higher the maternal education, the lower the incidence rate is of hepatoblastoma (17).

\section{GENETIC SYNDROMES}

Although most hepatoblastomas are sporadic (18), hepatoblastoma was reported to be closely related to genetic syndromes, including familial adenomatous polyposis (FAP), Beckwith-Wiedemann syndrome (BWS), and trisomy 18 (19-21).

The association between FAP and hepatoblastoma was originally perceived in 1983 (22). Mutation of the APC was detected in hepatoblastoma patients with a family history of FAP (23). APC mutation was also found in other cancers, including gastric and colorectal cancer $(24,25)$. However, in subsequent research, Harvey et al. demonstrated that no APC mutation was found in sporadic hepatoblastoma (26). BWS, an overgrowth syndrome associated with alteration of genomic imprinting on chromosome 11p15.5, is characterized by macroglossia, high birth weight, overgrowth of abdominal organs, and neonatal hypoglycemia $(27,28)$. Comparing with children without BWS, the relative rate of hepatoblastoma among children with BWS was 2,280 (95\% CI: 928-11,656) (29). Trisomy 18, regarded as a fatal disease, affects approximately 1 per 6,000 newborns (30). The correlation between trisomy 18 and hepatoblastoma has been reported in several previous studies (21,31-33). Tomlinson et al. raised an interesting point that the hepatoblastoma cases with trisomy 18 almost were females, and this situation was contrary to hepatoblastoma with higher prevalence in males (34). In addition to the syndromes mentioned above, some genetic syndromes have been reported, including Prader-Willi syndrome, and SimpsonGolabi-Behmel syndrome (35-37) (Table 1). However, these cases are too rare to consider as a close risk factor for hepatoblastoma.

\section{RISK FACTORS}

Although the etiology of hepatoblastoma remains unclear, some risk factors have been identified (Table 2). The United Kingdom Childhood Cancer Study (UKCCS) reported that parental
TABLE 1 | Genetic syndromes of hepatoblastoma.

\begin{tabular}{lclll}
\hline Syndrome & Chromosome & Correlated gene & Relative risk & Reference \\
\hline FAP & $5 q 21$ & APC & $750-7,500$ & $(38)$ \\
BWS & $11 \mathrm{p} 15.5$ & IGF2-H19 & 2280 & $(29)$ \\
Trisomy 18 & 18 & $/$ & Unknown & $(21)$ \\
SGBS & Xq26 & GPC3 & Unknown & $(37)$ \\
PWS & $46 \times Y$ del (15) & $/$ & Unknown & $(36)$ \\
& $(q 11, q 13)$ & & & \\
\hline
\end{tabular}

FAP, familial adenomatous polyposis; BWS, Beckwith-Wiedemann syndrome; SGBS, Simpson-Golabi-Behmel syndrome; PWS, Prader-Willi syndrome.

smoking is a risk factor for hepatoblastoma ( $\mathrm{OR}=4.74,95 \%$ CI:1.68-13.35), although only 28 hepatoblastoma patients were recruited in this study (39). The result of subsequent studies from the United States and China also supported this conclusion (40, 41). In 2009, parental smoking has been declared as the significant high-risk factor for hepatoblastoma by the International Agency for Research on Cancer (47).

As mentioned above, overgrowth syndrome is firmly correlated with hepatoblastoma; not only that, premature birth and very low birth weight (VLBW, $<1,500 \mathrm{~g}$ ) is closely associated with hepatoblastoma. The data analyzation, which was from California's population-based cancer registry, indicated that hepatoblastoma risk is remarkably increase in VLBW children ( $\mathrm{OR}=50.57$, 95\% CI: 6.59387.97) (42). Although the ORs were diverse in different regions, whole ORs were greater than one and proved VLBW was an obvious risk factor for hepatoblastoma $(15,40,43)$.

Janitz et al. had confirmed that maternal and paternal occupational exposures to paints were etiologically relevant to hepatoblastoma (44). Other studies indicated that parental occupational exposures to wood dust, metal fumes, and petroleum products also could be the risk factors $(45,46)$. On account of the rarity of hepatoblastoma, the investigations of risk factors are relatively limited.

\section{SINGLE NUCLEOTIDE POLYMORPHISMS WITH HEPATOBLASTOMA}

In the late 1990s, research showed that the existence of mutation of CTNNB1 ( $\beta$-catenin gene) might lead to $\beta$-catenin

TABLE 2 | Risk factors of hepatoblastoma.

\begin{tabular}{llccc}
\hline Risk factors & $\begin{array}{c}\text { Location/ } \\
\text { category }\end{array}$ & OR & 95\% Cl & Reference \\
\hline Parental smoking & UK & 4.74 & $1.68-13.35$ & $(39)$ \\
& China & 2.9 & $1.1-4.2$ & $(40)$ \\
\multirow{4}{*}{ VLBW } & US & 2.69 & $1.18-6.13$ & $(41)$ \\
& US & 50.57 & $6.59-387.97$ & $(42)$ \\
& China & 26.0 & $14.0-65.7$ & $(40)$ \\
Parental & Japan & 15.6 & $7.6-31.1$ & $(43)$ \\
occupational exposure & Nordic countries & 9.5 & $2.3-38.2$ & $(15)$ \\
& Paints & 1.71 & $1.04-2.81$ & $(44)$ \\
& & & & \\
& Wood dust & 2.41 & $0.99-5.88$ & $(45)$ \\
& Metal fumes & 8.0 & $1.5-148.4$ & $(46)$ \\
& Petroleum & 2.3 & $1.2-4.6$ & $(46)$
\end{tabular}


accumulation, resulting in the development of hepatoblastoma (48). Some pathways related to hepatoblastoma molecular mechanisms were detected, including the well-studied Wnt/ $\beta$ catenin and MYC pathways (49). However, research on the molecular basis in hepatoblastoma was limited. It is urgent to identify early diagnostic molecular-genetic markers for timely and valid therapeutic choices.

Single nucleotide polymorphisms (SNPs), comparing with rare gene mutation, were identified to abundantly exist in human genome by Human Genome Project (50). The susceptibilities and pathogenesis of disease and genetic heterogeneity are tightly correlated with SNPs (51-54). When present in non-coding regions, SNPs are regarded as critical genetic markers and can regulate protein expression (55). SNPs have been generally applied in the research of etiology's exploration, treatment of disease, and prognosis assessment. Comparing to other solid tumors in children, studies of the association between hepatoblastoma and gene polymorphism are relatively few. Here we summarized the significant hepatoblastoma susceptibility SNPs in Table 3.

\section{Cancer-Related Genes}

Pakakasama et al. addressed the association between myeloperoxidase (MPO) promotor gene polymorphism located on chromosome 17q23 and hepatoblastoma in 2003 (56). They demonstrated that MPO-463 G>A was associated with the reduced susceptibility of hepatoblastoma. Their study represents the first case-control study regarding genetic polymorphism and hepatoblastoma risk, although the cases were only less than 100. The significant roles of MPO-463 $\mathrm{G}>\mathrm{A}$ polymorphism were also reported in other cancers, including cervical, lung, breast, and bladder cancer (63-66). MPO is an oxidative enzyme located in neutrophils and monocytes. It can catalyze an oxidation reaction to generate hypochlorous acid $(\mathrm{HOCl})$, which is involved in DNA damage and inhibition of DNA repair (67). Carrying G/A or A/A genotype affects the expression of MPO and reduces the generation of oxygen radicals to decrease the risk of cancer.

In the following year, Pakakasama et al. conducted another study to elaborate that CCND1 gene rs9344 G>A polymorphism affecting gene splicing was associated with the age of onset of hepatoblastoma (57). CCND1 was identified as the core gene in the $\beta$-catenin/LEF pathway, which is relevant to hepatoblastoma's development $(68,69)$. The same as the study mentioned above, the cases of these two studies were less than 100 , which limited the reliability of the statistical result in the subgroups. In order to affirm these conclusions, study subjects are supposed to enlarge in a future study.

\section{RAS Gene}

More than a decade after that, the progression of studies about SNPs and hepatoblastoma was stagnant. In 2019, the third study, a relatively large-scale case-control study that recruited 213 cases in Chinese children, was conducted by our research group (70). As a famous oncogenic role, the RAS gene (KRAS, NRAS, and $H R A S)$ is commonly mutated in human cancers (71-73). However, in this study, we regrettably identified that one NRAS polymorphism and three KRAS polymorphisms do not correlate with hepatoblastoma susceptibility.

\section{Long Non-coding RNAs (IncRNAs)}

LncRNAs, with over 200 nucleotides in length and involved in diverse gene regulation, account for a large number of ncRNAs (74). Various studies have verified that lncRNAs play a vital role in transcription processes, regulation of cellular contexts, assembly of protein, tumor suppressor dysregulation, and other crucial biological function (75-80). LINC00673, located on chromosome $17 \mathrm{q} 24.3$, has been reported as an oncogene in diverse cancers (81-83). Childs et al. performed a genomewide association study (GWAS) to confirm that LINC00673 rs11655237 polymorphism is associated with pancreatic cancer susceptibility (84). Considering the involvement of LINC00673 in the occurrence and development of diverse cancers while there were no previous studies linking LINC00673 to hepatoblastoma, our research group conducted a case-control study selecting this polymorphism and confirmed that the LINC00673 rs11655237 C $>\mathrm{T}$ polymorphism may be correlated with hepatoblastoma susceptibility. In the stratified analysis, significant result was also found in the subgroup of clinical stages III+IV (58). The patients carrying this SNP seemed to tend to suffer severe hepatoblastoma. The conjecture based on the statistical result needs further validation.

TABLE 3 | Summary of hepatoblastoma susceptibility SNPS.

\begin{tabular}{|c|c|c|c|c|c|}
\hline Chromosome & Variant & Candidate gene & Alternate allele & Effect & Reference \\
\hline $17 q 23$ & G-463-A & MPO & $G>A$ & Protective factor & (56) \\
\hline $11 q 13$ & rs9344 & CCND1 & $G>A$ & Risk factor & (57) \\
\hline $17 q 24.3$ & rs11655237 & LINC00673 & $\mathrm{C}>\mathrm{T}$ & Risk factor & (58) \\
\hline $12 q 15$ & rs968697 & HMGA2 & $\mathrm{T}>\mathrm{C}$ & Risk factor & (52) \\
\hline $11 \mathrm{p} 15.5$ & rs2839698 & $\mathrm{H} 19$ & $\mathrm{G}>\mathrm{A}$ & Risk factor & (59) \\
\hline $11 p 15.5$ & rs3024270 & $\mathrm{H} 19$ & $\mathrm{C}>\mathrm{G}$ & Risk factor & (59) \\
\hline $11 p 15.5$ & rs217727 & $\mathrm{H} 19$ & $G>A$ & Protective factor & (59) \\
\hline $20 q 13$ & rs6090311 & YTHDF1 & $A>G$ & Protective factor & (60) \\
\hline $6 q 21$ & rs9404590 & LIN28B & $\mathrm{T}>\mathrm{G}$ & Risk factor & (61) \\
\hline $6 q 21$ & rs314276 & LIN28B & $\mathrm{C}>\mathrm{A}$ & Risk factor & (61) \\
\hline $6 q 25$ & rs7766006 & WTAP & $\mathrm{G}>\mathrm{T}$ & Protective factor & (62) \\
\hline $3 p 25.3$ & rs23795 & hOGG1 & $A>G$ & Risk factor & \\
\hline
\end{tabular}


LncRNA H19 gene, a maternally imprinted gene, is located on chromosome 11p5.5 and highly expressed during the stage of embryonic development $(85,86)$. H19 plays a vital role in tumorigenesis and the development of malignant tumors via regulation of transcription (87). Tan et al. identified that rs2839698 G>A and rs3024270 C>G, which decreased long non-coding RNA MRPL23 antisense RNA 1 (MRPL23-AS1) expression, were significantly correlated with increased hepatoblastoma risk. In contrast, rs217727 G>A increased MRPL23-AS1 expression to reduced hepatoblastoma risk in the Han population (59). Carrying GGG and AGG haplotypes (order rs2839698, rs3024270, rs217727), children have a tendency to suffer hepatoblastoma. These three polymorphisms were reported to affect the folding structures of H19 mRNA (88). These results revealed that even though SNPs are in the same gene, their effects of hepatoblastoma may be different. These SNPs are expected to be the biomarkers of early diagnosis of hepatoblastoma. However, the functions of H19 polymorphism in hepatoblastoma still need to be further validated.

\section{LIN28}

LIN28A and LIN28B are two paralogs of LIN28, located in chromosome $1 \mathrm{p} 36.11$ and $6 \mathrm{q} 21$, respectively (89). They can bind to the target RNAs, involved separately or jointly in human development and metabolism, to affect cancer occurrence via inhibition of let-7 miRNA (90). Yang et al. enrolled 275 hepatoblastoma cases and 1,018 healthy controls to prove LIN28B SNPs (rs94904590 T>G and rs314276 C >A) could increase the risk of hepatoblastoma (61). The LIN28A SNP (rs3811464 G>A) in hepatoblastoma affected hepatoblastoma in a low-penetrating manner because the significant result was only found in the stratified analysis (91). Four LIN28A SNPs (rs3811464 G>A, rs3811463 T>C, rs34787247 G>A, and rs11247957 G>A) were analyzed in hepatoblastoma, neuroblastoma, and Wilms tumor. Nevertheless, the association between the same SNPs with different malignant tumors is diverse (91-93). Interestingly, these findings suggested that the effects of $L I N 28 \mathrm{~A}$ polymorphisms were specific in a specific cancer.

\section{HMGA2}

HMGA2, a member of the high mobility group (HMG) proteins family, carries a typical functional sequence motif named AT-hooks (94). HMGA2 regulates gene transcription in a modification of chromatin construction way (95). Moreover, it is mainly expressed in embryonic stem cells during embryogenesis rather than in adult tissue cells (96). Many studies identified that aberrant HMGA2 expression is associated with diverse cancer (94). Li et al. detected that HMGA2 rs968697 T>C polymorphism was related to hepatoblastoma susceptibility in Chinese children (52).

\section{Base Excision Repair Pathway Genes}

DNA damage is common in humans, and DNA repair systems maintain the stability and integrity of DNA. If the damaged DNA is not repaired, genomic instability may eventually evolve into tumorigenesis (97). The base excision repair (BER) pathway is a critical part of DNA repair systems (98). Zhuo et al. conducted a case-control study exploring the relationship between six BER pathway genes (PARP1, hOGG1, FEN1, APEX1, LIG3, and $X R C C 1)$ and hepatoblastoma. hOGG1 gene rs293795 A $>\mathrm{G}$ was significantly correlated with hepatoblastoma risk (99).

\section{Other Genes}

As a tumor suppressor gene studied widely, TP53 is located on human chromosome 17p13.1 and plays a vital role in apoptosis and tumorigenesis (100). Among genes, TP53 has the highest pertinence with human tumors. Aberrant expression and dysfunction of TP53 have been detected in various human tumor cases. The TP53 rs1042522 C>G polymorphism leads to an amino acid alteration (Arg to Pro) and therefore influences the susceptibility of various malignant tumors (101-103). Our research group conducted two studies to explore the association between TP53 rs1042522 C>G polymorphism and hepatoblastoma. The significant result could not be found in the first study, which enrolled 213 hepatoblastoma cases and 958 cancer-free controls (104). After enlarging the study subjects to 313 cases and 1,446 controls and adding the analysis of rs4938723 $\mathrm{T}>\mathrm{C}$ of $m i R-34 b / c$, Liu et al. did not observe any significant result either (105). CMYC is also a critical oncogene and reported that the expression of c-Myc increased in hepatoblastoma tissue (106). However, Yang et al. conducted a study in Chinese children and the result showed that CMYC rs4645943 and rs2070583 polymorphisms were not correlated with hepatoblastoma risk (107).

\section{N6-Methyladenosine $\left(\mathrm{m}^{6} \mathrm{~A}\right)$ Modification Genes}

Mainly occurring on the N6-position of adenosine, $\mathrm{m}^{6} \mathrm{~A}$ as an invertible epigenetic modification is prevalent in various eucaryotes (108). $\mathrm{m}^{6} \mathrm{~A}$ prefers appearing in 3'untranslated regions (3'UTRs), around termination codons, and within long internal exons (109). Although $\mathrm{m}^{6} \mathrm{~A}$ modification does not disturb base paring or coding, it was reported that it is involved in various RNA metabolism, including RNA expression, alternative splicing, and export, and therefore plays a critical role in tumor occurrence and development (110). Cui et al. demonstrated that the majority of $\mathrm{m}^{6} \mathrm{~A}$-related genes were overexpressed in hepatoblastoma tissues (111). However, at present, the research about $\mathrm{m}^{6} \mathrm{~A}$-related gene polymorphism in hepatoblastoma is few.

$\mathrm{m}^{6} \mathrm{~A}$ proteins can be divided into three categories, namely, "writers", "erasers", and "readers", which have the function of adding, removing, and recognizing, respectively (112). Methyltransferase-like 3 (METTL3) is a catalytic enzyme that, combined with methyltransferase-like 14 (METTL14), becomes a heterocomplex; and Wilms' tumor 1-associated protein (WTAP) as an assistant protein interacts with this heterocomplex (113). METTL3 was localized at nuclear speckles and was involved in mRNA splicing to regulate mRNA metabolism (110). Liu et al. demonstrated that high expression of METTL3 is the main factor of the aberration of $\mathrm{m}^{6} \mathrm{~A}$ and thereby promotes hepatoblastoma growth via the $\mathrm{Wnt} / \beta$-catenin pathway (114). Downregulation of METTL14 was demonstrated to correlate with the prognosis of hepatocellular carcinoma (47). In contrast, the expression of WTAP was significantly upregulated in hepatocellular carcinoma (115). $\mathrm{m}^{6} \mathrm{~A}$ demethylases, known as "erasers", include obesity-associated protein (FTO) and alkylation repair homolog protein 5 (ALKBH5). 
FTO and ALKBH5 are $\alpha$-ketoglutarate $(\alpha-\mathrm{KG})$ and Fe (II)dependent demethylases that remove the RNA $\mathrm{m}^{6} \mathrm{~A}$ modification (116). There are many kinds of "readers" enzymes including YTH domain-containing family (YTHDF1-3 and YTHDC1-2), HNRNPA2BFHU1, Mrb1, ELAVL1, IGF2BPs, and eIFs. "Writers", as binding proteins with the function of specific recognition, impact the gene expression after RNA transcription (117).

To our knowledge, only our research group investigated the relationship between $\mathrm{m}^{6} \mathrm{~A}$ modification core gene polymorphisms and hepatoblastoma risk. Several research results have been published. Enrolled 313 cases and 1,446 controls, a Chinese sevencenter case-control study was conducted. The WTAP rs7766006 G>T was significantly correlated with reduced hepatoblastoma risk. Preliminary annotation revealed that WTAP mRNA levels were upregulated in the liver in those who carried the rs7766006 T genotype (62). The YTHDF1 rs6090311 G allele was identified as a protective factor of hepatoblastoma, and expression quantitative trait loci (eQTL) analyses showed that rs6090311 A>G might affect the mRNA level (60). Similar studies detected the association between YTHDC1 and ALKBH5 polymorphism and hepatoblastoma. However, significant relationships between YTHDC1 rs2293596 $\mathrm{T}>\mathrm{C}$ and ALKBH5 rs8400 $\mathrm{G}>\mathrm{A}$ polymorphism and hepatoblastoma risk were observed in the subgroup of clinical stage III+IV in stratification analysis $(118,119)$, requiring further study to identify whether these SNPs correlate to the prognosis of hepatoblastoma. Results indicated that the $\mathrm{m}^{6} \mathrm{~A}$ gene SNPs might affect the $\mathrm{m}^{6} \mathrm{~A}$ modification and thereby influence the hepatoblastoma growth (Figure 1). Our research group has also conducted the study of METTL3 and METTL14 polymorphisms and hepatoblastoma risk, and these results await to be published.

Considering the importance of $\mathrm{m}^{6} \mathrm{~A}$ modification in malignant tumors, the studies of $\mathrm{m}^{6} \mathrm{~A}$ modification core gene polymorphisms and hepatoblastoma risk are still inadequate. Besides, the number of SNPs included in the research is not enough, and more SNPs are urging to be enrolled to analyze.

\section{DISCUSSION AND FUTURE DIRECTIONS}

The treatment approaches of hepatoblastoma mainly include chemotherapy, surgical resection, and liver transplantation. In order to avoid overtreatments and improve the efficiency of treatment, individualized approaches are needed to provide to patients with different conditions. According to the recommendation of International Childhood Liver Tumor Strategy Group (SIOPEL), patients with high risk are suggested to be given dose-dense cisplatin weekly (120). Up to now, only alpha-fetoprotein (AFP) is used as a biomarker in clinics (121). If more biomarkers are applied in clinical practice for early diagnosis, the survival rate could be vastly improved. We reviewed the development of molecular epidemiology of hepatoblastoma. Advances in genotyping technologies facilitate the measuring of polymorphisms in a mass of samples. The sample sizes are finite in previous studies. However, the sample sizes have been distinctly enlarged with the launching of multicenter studies.

There some limitations of the research that actually need to be addressed. In recent years, the study subjects mentioned above are all from the Han population. Therefore, the results could not be generalized to other ethnicities. The precise functional role of SNPs in hepatoblastoma still awaits to be explored. Besides, there are plenty of cancer-related gene polymorphisms waiting to be detected.

In the past decades, genome-wide association study (GWAS), which is a method of conducting high-throughput sequencing technologies to measure plenty of polymorphisms, has discovered abundant significant cancer-related loci to improve

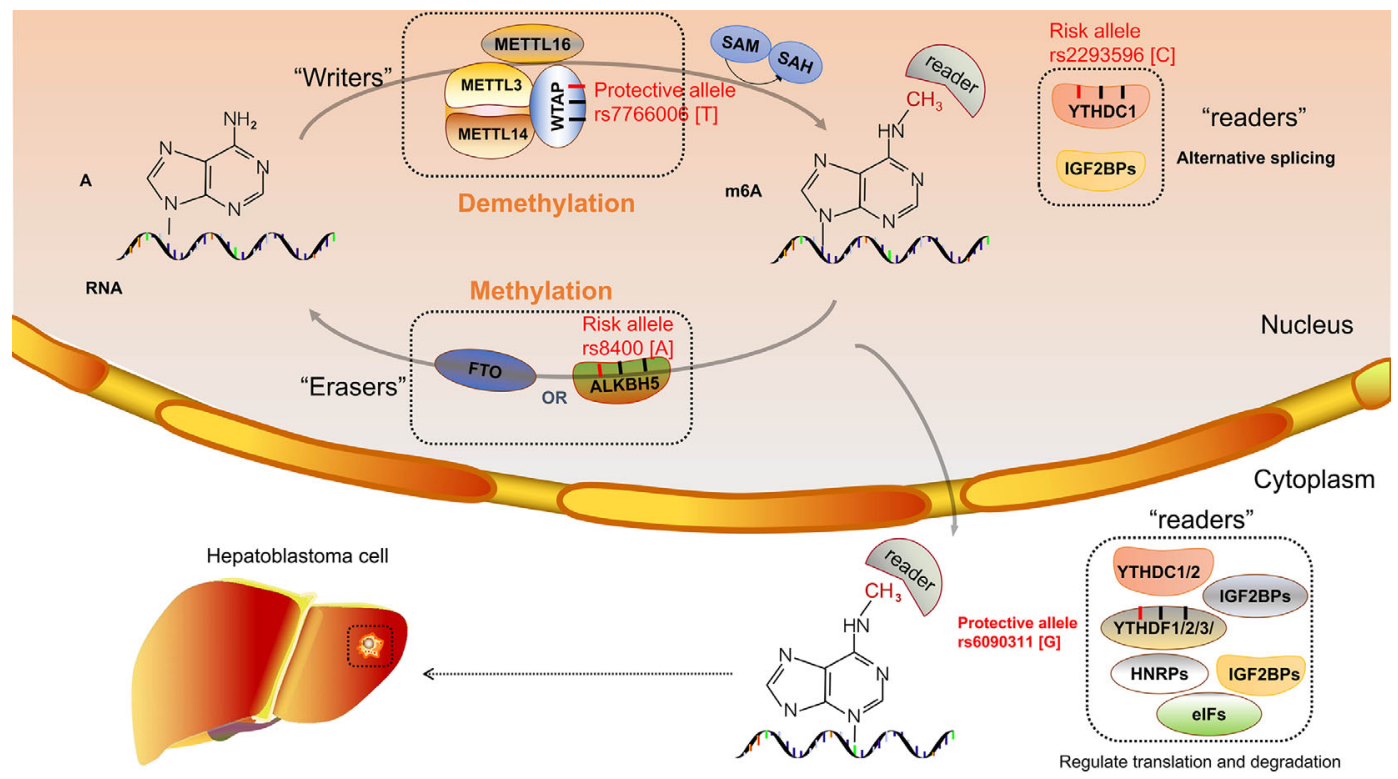

FIGURE 1 | N6-methyladenosine $\left(\mathrm{m}^{6} \mathrm{~A}\right)$ modification core gene polymorphisms and hepatoblastoma risk. 
the methods of genetic research $(122,123)$. This technology was applied in other childhood solid tumors such as neuroblastoma and has determined multiple disease-related loci $(124,125)$. GWAS needs a larger sample to apply in multistages and multicenters to ensure the reliability of the results. Based on the result that abundant SNPs were detected by GWAS, it is challenging to seek out the actual cancer-related loci and explain their biological function in hepatoblastoma. Identifying SNPs with important functionalities could be applied in prenatal screening to diminish birth defects. There is no doubt that the application of GWAS in hepatoblastoma is necessary. It will contribute to figuring out the biomarkers of hepatoblastoma and guiding people to understand hepatoblastoma's etiology to improve the prevention and treatment of hepatoblastoma.

\section{REFERENCES}

1. Sia D, Villanueva A, Friedman SL, Llovet JM. Liver Cancer Cell of Origin, Molecular Class, and Effects On Patient Prognosis. Gastroenterology (2017) 152(4):745-61. doi: 10.1053/j.gastro.2016.11.048

2. Kalish JM, Doros L, Helman LJ, Hennekam RC, Kuiper RP, Maas SM, et al. Surveillance Recommendations for Children With Overgrowth Syndromes and Predisposition to Wilms Tumors and Hepatoblastoma. Clin Cancer Res (2017) 23(13):e115-22. doi: 10.1158/1078-0432.CCR-17-0710

3. Musick SR, Smith M, Rouster AS, Babiker HM. Hepatoblastoma. In: StatPearls. Treasure Island (FL: StatPearls Publishing Copyright (C) 2021, StatPearls Publishing LLC (2021).

4. Feng J, Polychronidis G, Heger U, Frongia G, Mehrabi A, Hoffmann K. Incidence Trends and Survival Prediction of Hepatoblastoma in Children: A Population-Based Study. Cancer Commun (London England) (2019) 39 (1):62. doi: 10.1186/s40880-019-0411-7

5. Sivaprakasam P, Gupta AA, Greenberg ML, Capra M, Nathan PC. Survival and Long-Term Outcomes in Children With Hepatoblastoma Treated With Continuous Infusion of Cisplatin and Doxorubicin. J Pediatr Hematol/Oncol (2011) 33(6):e226-30. doi: 10.1097/MPH.0b013e31821f0eaf

6. Lake CM, Tiao GM, Bondoc AJ. Surgical Management of Locally-Advanced and Metastatic Hepatoblastoma. Semin Pediatr Surg (2019) 28(6):150856. doi: 10.1016/j.sempedsurg.2019.150856

7. Wiwanitkit V. Hepatitis Virus B is Not a Risk Factor in Hepatoblastoma Patients. Asian Pacific J Cancer Prev APJCP (2005) 6(2):213-4.

8. Ding SF, Michail NE, Habib NA. Genetic Changes in Hepatoblastoma. J Hepatol (1994) 20(5):672-5. doi: 10.1016/s0168-8278(05)80359-9

9. Spector LG, Birch J. The Epidemiology of Hepatoblastoma. Pediatr Blood Cancer (2012) 59(5):776-9. doi: 10.1002/pbc.24215

10. Hafberg E, Borinstein SC, Alexopoulos SP. Contemporary Management of Hepatoblastoma. Curr Opin Organ Transplant (2019) 24:113-7. doi: 10.1097/MOT.0000000000000618

11. Maris JM. Recent Advances in Neuroblastoma. New Engl J Med (2010) 362 (23):2202-11. doi: 10.1056/NEJMra0804577

12. Mahamdallie S, Yost S, Poyastro-Pearson E, Holt E, Zachariou A, Seal S, et al. Identification of New Wilms Tumour Predisposition Genes: An Exome Sequencing Study. Lancet Child Adolesc Health (2019) 3(5):322-31. doi: 10.1016/s2352-4642(19)30018-5

13. Linabery AM, Ross JA. Trends in Childhood Cancer Incidence in the U.S. (1992-2004). Cancer (2008) 112(2):416-32. doi: 10.1002/cncr.23169

14. Bao PP, Li K, Wu CX, Huang ZZ, Wang CF, Xiang YM, et al. [Recent Incidences and Trends of Childhood Malignant Solid Tumors in Shanghai, 2002-2010]. Zhonghua er ke za zhi = Chin J Pediatr (2013) 51(4):288-94.

15. de Fine Licht S, Schmidt LS, Rod NH, Schmiegelow K, Lähteenmäki PM, Kogner P, et al. Hepatoblastoma in the Nordic Countries. Int J Cancer (2012) 131(4):E555-61. doi: 10.1002/ijc.27351

16. Friedrich P, Itriago E, Rodriguez-Galindo C, Ribeiro K. Racial and Ethnic Disparities in the Incidence of Pediatric Extracranial Embryonal Tumors. J Natl Cancer Inst (2017) 109(10). doi: 10.1093/jnci/djx050

\section{AUTHOR CONTRIBUTIONS}

LM and ZZ: conceptualization and supervision. HC and QG: resources. HC: writing-original draft preparation. QG, HG, and LM: writing-review and editing. ZZ: project administration. LM and ZZ: funding acquisition. All authors contributed to the article and approved the submitted version.

\section{FUNDING}

This study was supported by grants from the National Natural Science Foundation of China (nos. 82002636, 82002635) and China Postdoctoral Science Foundation (nos. 2020T130132, 2020M682668).

17. Kehm RD, Spector LG, Poynter JN, Vock DM, Osypuk TL. Socioeconomic Status and Childhood Cancer Incidence: A Population-Based Multilevel Analysis. Am J Epidemiol (2018) 187(5):982-91. doi: 10.1093/aje/kwx322

18. Calvisi DF, Solinas A. Hepatoblastoma: Current Knowledge and Promises From Preclinical Studies. Trans Gastroenterol Hepatol (2020) 5:42. doi: $10.21037 / \operatorname{tgh} .2019 .12 .03$

19. Trobaugh-Lotrario AD, López-Terrada D, Li P, Feusner JH. Hepatoblastoma in Patients With Molecularly Proven Familial Adenomatous Polyposis: Clinical Characteristics and Rationale for Surveillance Screening. Pediatr Blood Cancer (2018) 65(8):e27103. doi: 10.1002/pbc.27103

20. Hamada Y, Takada K, Fukunaga S, Hioki K. Hepatoblastoma Associated With Beckwith-Wiedemann Syndrome and Hemihypertrophy. Pediatr Surg Int (2003) 19(1-2):112-4. doi: 10.1007/s00383-002-0734-2

21. Pereira EM, Marion R, Ramesh KH, Kim JS, Ewart M, Ricafort R. Hepatoblastoma in a Mosaic Trisomy 18 Patient. J Pediatr Hematol/Oncol (2012) 34(4):e145-8. doi: 10.1097/MPH.0b013e3182459ee8

22. Kingston JE, Herbert A, Draper GJ, Mann JR. Association Between Hepatoblastoma and Polyposis Coli. Arch Dis Childhood (1983) 58 (12):959-62. doi: 10.1136/adc.58.12.959

23. Giardiello FM, Petersen GM, Brensinger JD, Luce MC, Cayouette MC, Bacon J, et al. Hepatoblastoma and APC Gene Mutation in Familial Adenomatous Polyposis. Gut (1996) 39(6):867-9. doi: 10.1136/gut.39.6.867

24. Fodde R. The APC Gene in Colorectal Cancer. Eur J Cancer (2002) 38 (7):867-71. doi: 10.1016/s0959-8049(02)00040-0

25. Fang DC, Luo YH, Yang SM, Li XA, Ling XL, Fang L. Mutation Analysis of APC Gene in Gastric Cancer With Microsatellite Instability. World J Gastroenterol (2002) 8(5):787-91. doi: 10.3748/wjg.v8.i5.787

26. Harvey J, Clark S, Hyer W, Hadzic N, Tomlinson I, Hinds R. Germline APC Mutations are Not Commonly Seen in Children With Sporadic Hepatoblastoma. J Pediatr Gastroenterol Nutr (2008) 47(5):675-7. doi: 10.1097/MPG.0b013e318174e808

27. Kim SY, Jung SH, Kim MS, Han MR, Park HC, Jung ES, et al. Genomic Profiles of a Hepatoblastoma From a Patient With Beckwith-Wiedemann Syndrome With Uniparental Disomy on Chromosome 11p15 and Germline Mutation of APC and PALB2. Oncotarget (2017) 8(54):91950-7. doi: 10.18632/oncotarget.20515

28. Weksberg R, Shuman C, Beckwith JB, Weksberg R. Beckwith-Wiedemann Syndrome. Eur J Hum Genet (2010) 18(1):1-14. doi: 10.1038/ejhg. 2009.106

29. DeBaun MR, Tucker MA. Risk of Cancer During the First Four Years of Life in Children From The Beckwith-Wiedemann Syndrome Registry. J Pediatr (1998) 132(3 Pt 1):398-400. doi: 10.1016/s0022-3476(98)70008-3

30. Wang TJ, Li YY, Wu WJ, Lin CK, Wang CK, Wang CY, et al. Dandy-Walker Syndrome With Duplex Kidney Abnormalities in Trisomy 18 - A Rare Case Report. Taiwanese J Obstetrics Gynecol (2017) 56(5):697-9. doi: 10.1016/ j.tjog.2017.08.022

31. Ahmad N, Wheeler K, Stewart H, Campbell C. Hepatoblastoma in a Mosaic Trisomy 18 Child With Hemihypertrophy. BMJ Case Rep (2016) 2016. doi: $10.1136 /$ bcr-2015-211380 
32. Inoue A, Suzuki R, Urabe K, Kawamura Y, Masuda M, Kishi K, et al. Therapeutic Experience With Hepatoblastoma Associated With Trisomy 18. Pediatr Blood Cancer (2018) 65(8):e27093. doi: 10.1002/pbc.27093

33. Lucas DJ, Rubinstein J, Gosain A, Tiao G, Head T, Pratap JN, et al. Surgical and Anesthetic Management for Hepatectomy in Two Pediatric Patients With Trisomy 18, Pulmonary Hypertension, and Hepatoblastoma. Pediatr Blood Cancer (2019) 66(6):e27678. doi: 10.1002/pbc.27678

34. Tomlinson GE, Kappler R. Genetics and Epigenetics of Hepatoblastoma. Pediatr Blood Cancer (2012) 59(5):785-92. doi: 10.1002/pbc.24213

35. Ozawa MG, Cooney T, Rangaswami A, Hazard FK. Synchronous Hepatoblastoma, Neuroblastoma, and Cutaneous Capillary Hemangiomas: A Case Report. Pediatr Dev Pathol (2016) 19(1):74-9. doi: 10.2350/14-111573-cr.1

36. Takayasu H, Motoi T, Kanamori Y, Kitano Y, Nakanishi H, Tange T, et al. Two Case Reports of Childhood Liver Cell Adenomas Harboring BetaCatenin Abnormalities. Hum Pathol (2002) 33(8):852-5. doi: 10.1053/ hupa.2002.125771

37. Mateos ME, Beyer K, López-Laso E, Siles JL, Pérez-Navero JL, Peña MJ, et al. Simpson-Golabi-Behmel Syndrome Type 1 and Hepatoblastoma in a Patient With a Novel Exon 2-4 Duplication of the GPC3 Gene. Am J Med Genet Part A (2013) 161a(5):1091-5. doi: 10.1002/ajmg.a.35738

38. Aretz S, Koch A, Uhlhaas S, Friedl W, Propping P, von Schweinitz D, et al. Should Children at Risk for Familial Adenomatous Polyposis be Screened for Hepatoblastoma and Children With Apparently Sporadic Hepatoblastoma be Screened for APC Germline Mutations? Pediatr Blood Cancer (2006) 47 (6):811-8. doi: 10.1002/pbc.20698

39. Pang D, McNally R, Birch JM. Parental Smoking and Childhood Cancer: Results From the United Kingdom Childhood Cancer Study. Br J Cancer (2003) 88(3):373-81. doi: 10.1038/sj.bjc.6600774

40. Pu CL, Guo CB, Jin XQ, Deng C, Zhang MM, Li YC, et al. [Retrospective Analysis of Maternal and Infant Birth Features of Hepatoblastoma Patients]. Zhonghua gan zang bing za zhi = Zhonghua Ganzangbing Zazhi = Chin J Hepatol (2009) 17(6):459-61.

41. Sorahan T, Lancashire RJ. Parental Cigarette Smoking and Childhood Risks of Hepatoblastoma: OSCC Data. Br J Cancer (2004) 90(5):1016-8. doi: $10.1038 /$ sj.bjc.6601651

42. Reynolds P, Urayama KY, Von Behren J, Feusner J. Birth Characteristics and Hepatoblastoma Risk in Young Children. Cancer (2004) 100(5):1070-6. doi: $10.1002 / \mathrm{cncr} .20061$

43. Tanimura M, Matsui I, Abe J, Ikeda H, Kobayashi N, Ohira M, et al. Increased Risk of Hepatoblastoma Among Immature Children With a Lower Birth Weight. Cancer Res (1998) 58(14):3032-5.

44. Janitz AE, Ramachandran G, Tomlinson GE, Krailo M, Richardson M, Spector L. Maternal and Paternal Occupational Exposures and Hepatoblastoma: Results From the HOPE Study Through the Children's Oncology Group. J Exposure Sci Environ Epidemiol (2017) 27(4):359-64. doi: $10.1038 /$ jes.2017.1

45. Volk J, Heck JE, Schmiegelow K, Hansen J. Parental Occupational Organic Dust Exposure and Selected Childhood Cancers in Denmark 1968-2016. Cancer Epidemiol (2020) 65:101667. doi: 10.1016/j.canep.2020.101667

46. Buckley JD, Sather H, Ruccione K, Rogers PC, Haas JE, Henderson BE, et al. A Case-Control Study of Risk Factors for Hepatoblastoma. A Report From the Childrens Cancer Study Group. Cancer (1989) 64(5):1169-76. doi: 10.1002/ 1097-0142(19890901)64:5<1169::aid-cncr2820640534>3.0.c0;2-i

47. Ma JZ, Yang F, Zhou CC, Liu F, Yuan JH, Wang F, et al. METTL14 Suppresses the Metastatic Potential of Hepatocellular Carcinoma by Modulating N(6) -Methyladenosine-Dependent Primary MicroRNA Processing. Hepatology (2017) 65(2):529-43. doi: 10.1002/hep.28885

48. Bläker H, Hofmann WJ, Rieker RJ, Penzel R, Graf M, Otto HF. Beta-Catenin Accumulation and Mutation of the CTNNB1 Gene in Hepatoblastoma. Genes Chromosomes Cancer (1999) 25(4):399-402. doi: 10.1002/(SICI)10982264(199908)25:4<399::AID-GCC14>3.0.CO;2-X

49. Shen G, Shen H, Zhang J, Yan Q, Liu H. DNA Methylation in Hepatoblastoma-A Literature Review. Ital J Pediatr (2020) 46(1):113. doi: 10.1186/s13052-020-00877-6

50. Shah T, Joshi K. Analysis of FOXO3a Gene Polymorphism Associated With Asthma. Methods Mol Biol (Clifton NJ) (2019) 1890:259-66. doi: 10.1007/ 978-1-4939-8900-3_22
51. Liu X, Han Z, Yang C. Associations of microRNA Single Nucleotide Polymorphisms and Disease Risk and Pathophysiology. Clin Genet (2017) 92(3):235-42. doi: 10.1111/cge.12950

52. Yang S, Zheng Y, Zhou L, Jin J, Deng Y, Yao J, et al. miR-499 Rs3746444 and miR-196a-2 Rs11614913 Are Associated With the Risk of Glioma, But Not the Prognosis. Mol Ther Nucleic Acids (2020) 22:340-51. doi: 10.1016/ j.omtn.2020.08.038

53. Zhou L, Dong S, Deng Y, Yang P, Zheng Y, Yao L, et al. GOLGA7 Rs11337, a Polymorphism at the MicroRNA Binding Site, Is Associated With Glioma Prognosis. Mol Ther Nucleic Acids (2019) 18:56-65. doi: 10.1016/ j.omtn.2019.08.006

54. Yang Y, Shu X, Shu XO, Bolla MK, Kweon SS, Cai Q, et al. Re-Evaluating Genetic Variants Identified in Candidate Gene Studies of Breast Cancer Risk Using Data From Nearly 280,000 Women of Asian and European Ancestry. EBioMedicine (2019) 48:203-11. doi: 10.1016/j.ebiom.2019.09.006

55. Kim S, Misra A. SNP Genotyping: Technologies and Biomedical Applications. Annu Rev Biomed Eng (2007) 9:289-320. doi: 10.1146/ annurev.bioeng.9.060906.152037

56. Pakakasama S, Chen TT, Frawley W, Muller C, Douglass EC, Tomlinson GE. Myeloperoxidase Promotor Polymorphism and Risk of Hepatoblastoma. Int J Cancer (2003) 106(2):205-7. doi: 10.1002/ijc.11191

57. Pakakasama S, Chen TT, Frawley W, Muller CY, Douglass EC, Lee R, et al. CCND1 Polymorphism and Age of Onset of Hepatoblastoma. Oncogene (2004) 23:4789-92. doi: 10.1038/sj.onc.1207499

58. Yang T, Li J, Wen Y, Tan T, Yang J, Pan J, et al. LINC00673 Rs11655237 C>T Polymorphism Impacts Hepatoblastoma Susceptibility in Chinese Children. Front Genet (2019) 10:506. doi: 10.3389/fgene.2019.00506

59. Tan T, Li J, Wen Y, Zou Y, Yang J, Pan J, et al. Association Between lncRNAH19 Polymorphisms and Hepatoblastoma Risk in an Ethic Chinese Population. J Cell Mol Med (2021) 25(2):742-50. doi: 10.1111/jcmm.16124

60. Luo Z, Li G, Wang M, Zhu J, Yang Z, Li Y, et al. YTHDF1 Rs6090311 a>G Polymorphism Reduces Hepatoblastoma Risk: Evidence From a SevenCenter Case-Control Study. J Cancer (2020) 11(17):5129-34. doi: 10.7150/ jca.46120

61. Yang Z, Deng Y, Zhang K, Bai Y, Zhu J, Zhang J, et al. LIN28B Gene Polymorphisms Modify Hepatoblastoma Susceptibility in Chinese Children. J Cancer (2020) 11(12):3512-8. doi: 10.7150/jca.42798

62. Zhuo ZJ, Hua RX, Chen Z, Zhu J, Wang M, Yang Z, et al. WTAP Gene Variants Confer Hepatoblastoma Susceptibility: A Seven-Center CaseControl Study. Mol Ther Oncol (2020) 18:118-25. doi: 10.1016/ j.omto.2020.06.007

63. Hung RJ, Boffetta P, Brennan P, Malaveille C, Gelatti U, Placidi D, et al. Genetic Polymorphisms of MPO, COMT, MnSOD, NQO1, Interactions With Environmental Exposures and Bladder Cancer Risk. Carcinogenesis (2004) 25(6):973-8. doi: 10.1093/carcin/bgh080

64. Lin SC, Chou YC, Wu MH, Wu CC, Lin WY, Yu CP, et al. Genetic Variants of Myeloperoxidase and Catechol-O-Methyltransferase and Breast Cancer Risk. Eur J Cancer Prev (2005) 14(3):257-61. doi: 10.1097/00008469200506000-00010

65. Shi X, Li B, Yuan Y, Chen L, Zhang Y, Yang M, et al. The Possible Association Between the Presence of an MPO -463 G > A (Rs2333227) Polymorphism and Cervical Cancer Risk. Pathol Res Pract (2018) 214 (8):1142-8. doi: 10.1016/j.prp.2018.05.018

66. Kantarci OH, Lesnick TG, Yang P, Meyer RL, Hebrink DD, McMurray CT, et al. Myeloperoxidase $-463(\mathrm{G}->\mathrm{A})$ Polymorphism Associated With Lower Risk of Lung Cancer. Mayo Clin Proc (2002) 77(1):17-22. doi: 10.4065/ 77.1.17

67. Ohnishi S, Murata M, Kawanishi S. DNA Damage Induced by Hypochlorite and Hypobromite With Reference to Inflammation-Associated Carcinogenesis. Cancer Lett (2002) 178(1):37-42. doi: 10.1016/s0304-3835 (01)00812-6

68. Govatati S, Singamsetty GK, Nallabelli N, Malempati S, Rao PS, Madamchetty VK, et al. Contribution of Cyclin D1 (CCND1) and ECadherin (CDH1) Alterations to Colorectal Cancer Susceptibility: A CaseControl Study. Tumour Biol (2014) 35(12):12059-67. doi: 10.1007/s13277014-2505-9

69. Takayasu H, Horie H, Hiyama E, Matsunaga T, Hayashi Y, Watanabe Y, et al. Frequent Deletions and Mutations of the Beta-Catenin Gene are 
Associated With Overexpression of Cyclin D1 and Fibronectin and Poorly Differentiated Histology in Childhood Hepatoblastoma. Clin Cancer Res (2001) 7(4):901-8.

70. Yang T, Wen Y, Li J, Tan T, Yang J, Pan J, et al. NRAS and KRAS Polymorphisms are Not Associated With Hepatoblastoma Susceptibility in Chinese Children. Exp Hematol Oncol (2019) 8:11. doi: 10.1186/s40164-0190135-z

71. Chen S, Li F, Xu D, Hou K, Fang W, Li Y. The Function of RAS Mutation in Cancer and Advances in its Drug Research. Curr Pharm Design (2019) 25 (10):1105-14. doi: 10.2174/1381612825666190506122228

72. Meng L, Liu S, Liu F, Sang M, Ju Y, Fan X, et al. ZEB1-Mediated Transcriptional Upregulation of Circwwc3 Promotes Breast Cancer Progression Through Activating Ras Signaling Pathway. Mol Ther Nucleic Acids (2020) 22:124-37. doi: 10.1016/j.omtn.2020.08.015

73. Wang H, Lv Q, Xu Y, Cai Z, Zheng J, Cheng X, et al. An Integrative Pharmacogenomics Analysis Identifies Therapeutic Targets in KRASMutant Lung Cancer. EBioMedicine (2019) 49:106-17. doi: 10.1016/ j.ebiom.2019.10.012

74. Esteller M. Non-Coding RNAs in Human Disease. Nat Rev Genet (2011) 12 (12):861-74. doi: $10.1038 / \mathrm{nrg} 3074$

75. Gong J, Liu W, Zhang J, Miao X, Guo AY. IncRNASNP: A Database of SNPs in lncRNAs and Their Potential Functions in Human and Mouse. Nucleic Acids Res (2015) 43(Database issue):D181-6. doi: 10.1093/nar/gku1000

76. Tang JY, Lee JC, Chang YT, Hou MF, Huang HW, Liaw CC, et al. Long Noncoding RNAs-Related Diseases, Cancers, and Drugs. Scientific WorldJournal (2013) 2013:943539. doi: 10.1155/2013/943539

77. Tan YT, Lin JF, Li T, Li JJ, Xu RH, Ju HQ. LncRNA-Mediated Posttranslational Modifications and Reprogramming of Energy Metabolism in Cancer. Cancer Commun (London England) (2021) 41 (2):109-20. doi: 10.1002/cac2.12108

78. Yang Y, Deng X, Li Q, Wang F, Miao L, Jiang Q. Emerging Roles of Long Noncoding RNAs in Cholangiocarcinoma: Advances and Challenges. Cancer Commun (London England) (2020) 40(12):655-80. doi: 10.1002/ cac2.12109

79. Song H, Liu Y, Li X, Chen S, Xie R, Chen D, et al. Long Noncoding RNA CASC11 Promotes Hepatocarcinogenesis and HCC Progression Through EIF4A3-Mediated E2F1 Activation. Clin Transl Med (2020) 10(7):e220. doi: $10.1002 / \mathrm{ctm} 2.220$

80. Hua RX, Liu J, Fu W, Zhu J, Zhang J, Cheng J, et al. ALKBH5 Gene Polymorphisms and Wilms Tumor Risk in Chinese Children: A Five-Center Case-Control Study. J Clin Lab Anal (2020) 34(6):e23251. doi: 10.1002/ jcla.23251

81. Qiao K, Ning S, Wan L, Wu H, Wang Q, Zhang X, et al. LINC00673 is Activated by YY1 and Promotes the Proliferation of Breast Cancer Cells via the miR-515-5p/MARK4/Hippo Signaling Pathway. J Exp Clin Cancer Res CR (2019) 38(1):418. doi: 10.1186/s13046-019-1421-7

82. Zheng J, Huang X, Tan W, Yu D, Du Z, Chang J, et al. Pancreatic Cancer Risk Variant in LINC00673 Creates a miR-1231 Binding Site and Interferes With PTPN11 Degradation. Nat Genet (2016) 48(7):747-57. doi: 10.1038/ ng. 3568

83. Lu W, Zhang H, Niu Y, Wu Y, Sun W, Li H, et al. Long non-Coding RNA Linc00673 Regulated non-Small Cell Lung Cancer Proliferation, Migration, Invasion and Epithelial Mesenchymal Transition by Sponging miR-150-5p. Mol Cancer (2017) 16(1):118. doi: 10.1186/s12943-017-0685-9

84. Childs EJ, Mocci E, Campa D, Bracci PM, Gallinger S, Goggins M, et al. Common Variation at 2p13.3, 3q29, 7p13 and 17q25.1 Associated With Susceptibility to Pancreatic Cancer. Nat Genet (2015) 47(8):911-6. doi: $10.1038 / \mathrm{ng} .3341$

85. Verhaegh GW, Verkleij L, Vermeulen SH, den Heijer M, Witjes JA, Kiemeney LA. Polymorphisms in the H19 Gene and the Risk of Bladder Cancer. Eur Urol (2008) 54(5):1118-26. doi: 10.1016/j.eururo.2008.01.060

86. Tanos V, Prus D, Ayesh S, Weinstein D, Tykocinski ML, De-Groot N, et al. Expression of the Imprinted H19 Oncofetal RNA in Epithelial Ovarian Cancer. Eur J Obstetrics Gynecol Reprod Biol (1999) 85(1):7-11. doi: 10.1016/ s0301-2115(98)00275-9

87. Lecerf C, Le Bourhis X, Adriaenssens E. The Long non-Coding RNA H19: An Active Player With Multiple Facets to Sustain the Hallmarks of Cancer.
Cell Mol Life Sci CMLS (2019) 76(23):4673-87. doi: 10.1007/s00018-01903240-z

88. Li S, Hua Y, Jin J, Wang H, Du M, Zhu L, et al. Association of Genetic Variants in lncRNA H19 With Risk of Colorectal Cancer in a Chinese Population. Oncotarget (2016) 7(18):25470-7. doi: 10.18632/ oncotarget.8330

89. Wang H, Zhao Q, Deng K, Guo X, Xia J. Lin28: An Emerging Important Oncogene Connecting Several Aspects of Cancer. Tumour Biol (2016) 37 (3):2841-8. doi: 10.1007/s13277-015-4759-2

90. Balzeau J, Menezes MR, Cao S, Hagan JP. The LIN28/let-7 Pathway in Cancer. Front Genet (2017) 8:31. doi: 10.3389/fgene.2017.00031

91. Yang Z, Deng Y, Zhang K, Bai Y, Zhu J, Zhang J, et al. LIN28A Polymorphisms and Hepatoblastoma Susceptibility in Chinese Children. J Cancer (2021) 12(5):1373-8. doi: 10.7150/jca.52621

92. Zhuo Z, Fu W, Liu J, Cheng J, Zhou H, Zhang J, et al. LIN28A Gene Polymorphisms Confer Wilms Tumour Susceptibility: A Four-Centre CaseControl Study. J Cell Mol Med (2019) 23(10):7105-10. doi: 10.1111/ jcmm.14561

93. Hua RX, Zhuo Z, Ge L, Zhu J, Yuan L, Chen C, et al. LIN28A Gene Polymorphisms Modify Neuroblastoma Susceptibility: A Four-Centre CaseControl Study. J Cell Mol Med (2020) 24(1):1059-66. doi: 10.1111/ jcmm. 14827

94. Zhang BH, Yan LN, Yang JY. Pending Role of METTL14 in Liver Cancer. Hepatobil Surg Nutr (2019) 8(6):669-70. doi: 10.21037/hbsn.2019.10.16

95. Fedele M, Battista S, Kenyon L, Baldassarre G, Fidanza V, Klein-Szanto AJ, et al. Overexpression of the HMGA2 Gene in Transgenic Mice Leads to the Onset of Pituitary Adenomas. Oncogene (2002) 21(20):3190-8. doi: 10.1038/ sj.onc. 1205428

96. Zhou X, Benson KF, Ashar HR, Chada K. Mutation Responsible for the Mouse Pygmy Phenotype in the Developmentally Regulated Factor HMGIC. Nature (1995) 376(6543):771-4. doi: 10.1038/376771a0

97. Kumar N, Raja S, Van Houten B. The Involvement of Nucleotide Excision Repair Proteins in the Removal of Oxidative DNA Damage. Nucleic Acids Res (2020) 48(20):11227-43. doi: 10.1093/nar/gkaa777

98. Liu Y, Wilson SH. DNA Base Excision Repair: A Mechanism of Trinucleotide Repeat Expansion. Trends Biochem Sci (2012) 37(4):162-72. doi: 10.1016/j.tibs.2011.12.002

99. Zhuo Z, Lin A, Zhang J, Chen H, Li Y, Yang Z, et al. Genetic Variations in Base Excision Repair Pathway Genes and Risk of Hepatoblastoma: A SevenCenter Case-Control Study. Am J Cancer Res (2021) 11(3):849-57.

100. Xu H, El-Gewely MR. P53-Responsive Genes and the Potential for Cancer Diagnostics and Therapeutics Development. Biotechnol Annu Rev (2001) 7:131-64. doi: 10.1016/s1387-2656(01)07035-1

101. Icen-Taskin I, Irtegun-Kandemir S, Munzuroglu O. TP53 Rs1042522 Polymorphism and Early-Onset Breast Cancer. J Res Med Sci (2020) 25:25. doi: 10.4103/jrms.JRMS_506_19

102. Li Z, Weng H, Su R, Weng X, Zuo Z, Li C, et al. FTO Plays an Oncogenic Role in Acute Myeloid Leukemia as a N(6)-Methyladenosine RNA Demethylase. Cancer Cell (2017) 31:127-41. doi: 10.1016/j.ccell. 2016.11.017

103. Kamiza AB, Kamiza S, Singini MG, Mathew CG. Association of TP53 Rs1042522 With Cervical Cancer in the Sub-Saharan African Population: A Meta-Analysis. Trop Med Int Health TM IH (2020) 25(6):666-72. doi: $10.1111 /$ tmi.13397

104. Yang T, Wen Y, Li J, Yang J, Tan T, Pan J, et al. Association of the TP53 Rs1042522 C > G Polymorphism and Hepatoblastoma Risk in Chinese Children. J Cancer (2019) 10(15):3444-9. doi: 10.7150/jca.33063

105. Liu P, Zhuo ZJ, Zhu J, Yang Z, Xin Y, Li S, et al. Association of TP53 Rs1042522 C>G and miR-34b/C Rs4938723 T>C Polymorphisms With Hepatoblastoma Susceptibility: A Seven-Center Case-Control Study. J Gene Med (2020) 22(7):e3182. doi: 10.1002/jgm.3182

106. Ranganathan S, Tan X, Monga SP. Beta-Catenin and Met Deregulation in Childhood Hepatoblastomas. Pediatr Dev Pathol (2005) 8(4):435-47. doi: 10.1007/s10024-005-0028-5

107. Yang T, Wen Y, Li J, Tan T, Yang J, Pan J, et al. Association of CMYC Polymorphisms With Hepatoblastoma Risk. Transl Cancer Res (2020) 9 (2):849-55. doi: $10.21037 /$ tcr.2019.12.19 
108. Yue Y, Liu J, He C. RNA N6-Methyladenosine Methylation in PostTranscriptional Gene Expression Regulation. Genes Dev (2015) 29 (13):1343-55. doi: 10.1101/gad.262766.115

109. Ke S, Alemu EA, Mertens C, Gantman EC, Fak JJ, Mele A, et al. A Majority of M6a Residues are in the Last Exons, Allowing the Potential for 3' UTR Regulation. Genes Dev (2015) 29(19):2037-53. doi: 10.1101/gad.269415.115

110. Yang Y, Hsu PJ, Chen YS, Yang YG. Dynamic Transcriptomic M(6)A Decoration: Writers, Erasers, Readers and Functions in RNA Metabolism. Cell Res (2018) 28(6):616-24. doi: 10.1038/s41422-018-0040-8

111. Cui X, Wang Z, Li J, Zhu J, Ren Z, Zhang D, et al. Cross Talk Between RNA N6-Methyladenosine Methyltransferase-Like 3 and miR-186 Regulates Hepatoblastoma Progression Through Wnt/ $\beta$-Catenin Signalling Pathway. Cell Proliferation (2020) 53(3):e12768. doi: 10.1111/cpr.12768

112. He PC, He C. M(6) A RNA Methylation: From Mechanisms to Therapeutic Potential. EMBO J (2021) 40(3):e105977. doi: 10.15252/embj.2020105977

113. Zaccara S, Ries RJ, Jaffrey SR. Reading, Writing and Erasing mRNA Methylation. Nat Rev Mol Cell Biol (2019) 20(10):608-24. doi: 10.1038/ s41580-019-0168-5

114. Liu L, Wang J, Sun G, Wu Q, Ma J, Zhang X, et al. M(6)A mRNA Methylation Regulates CTNNB1 to Promote the Proliferation of Hepatoblastoma. Mol Cancer (2019) 18(1):188. doi: 10.1186/s12943-0191119-7

115. Chen Y, Peng C, Chen J, Chen D, Yang B, He B, et al. WTAP Facilitates Progression of Hepatocellular Carcinoma via M6a-HuR-Dependent Epigenetic Silencing of ETS1. Mol Cancer (2019) 18(1):127. doi: 10.1186/ s12943-019-1053-8

116. Gerken T, Girard CA, Tung YC, Webby CJ, Saudek V, Hewitson KS, et al. The Obesity-Associated FTO Gene Encodes a 2-Oxoglutarate-Dependent Nucleic Acid Demethylase. Sci (New York NY) (2007) 318(5855):1469-72. doi: $10.1126 /$ science. 1151710

117. Zhao Y, Shi Y, Shen H, Xie W. M(6)A-Binding Proteins: The Emerging Crucial Performers in Epigenetics. J Hematol Oncol (2020) 13(1):35. doi: 10.1186/s13045-020-00872-8

118. Chen H, Li Y, Li L, Zhu J, Yang Z, Zhang J, et al. YTHDC1 Gene Polymorphisms and Hepatoblastoma Susceptibility in Chinese Children: A Seven-Center Case-Control Study. J Gene Med (2020) 22(11):e3249. doi: 10.1002/jgm.3249
119. Ren H, Zhuo ZJ, Duan F, Li Y, Yang Z, Zhang J, et al. ALKBH5 Gene Polymorphisms and Hepatoblastoma Susceptibility in Chinese Children. J Oncol (2021) 2021:6658480. doi: 10.1155/2021/6658480

120. Zsiros J, Brugieres L, Brock P, Roebuck D, Maibach R, Zimmermann A, et al. Dose-Dense Cisplatin-Based Chemotherapy and Surgery for Children With High-Risk Hepatoblastoma (SIOPEL-4): A Prospective, Single-Arm, Feasibility Study. Lancet Oncol (2013) 14(9):834-42. doi: 10.1016/s14702045(13)70272-9

121. Maibach R, Roebuck D, Brugieres L, Capra M, Brock P, Dall'Igna P, et al. Prognostic Stratification for Children With Hepatoblastoma: The SIOPEL Experience. Eur J Cancer (Oxford Engl 1990) (2012) 48(10):1543-9. doi: 10.1016/j.ejca.2011.12.011

122. Kinnersley B, Houlston RS, Bondy ML. Genome-Wide Association Studies in Glioma. Cancer Epidemiol Biomarkers Prev (2018) 27(4):418-28. doi: 10.1158/1055-9965.Epi-17-1080

123. Benafif S, Kote-Jarai Z. Eeles RA. A Review of Prostate Cancer GenomeWide Association Studies (GWAS). Cancer Epidemiol Biomarkers Prev (2018) 27(8):845-57. doi: 10.1158/1055-9965.Epi-16-1046

124. He J, Zou Y, Wang T, Zhang R, Yang T, Zhu J, et al. Genetic Variations of GWAS-Identified Genes and Neuroblastoma Susceptibility: A Replication Study in Southern Chinese Children. Trans Oncol (2017) 10(6):936-41. doi: 10.1016/j.tranon.2017.09.008

125. Nguyen le B, Diskin SJ, Capasso M, Wang K, Diamond MA, Glessner J, et al. Phenotype Restricted Genome-Wide Association Study Using a GeneCentric Approach Identifies Three Low-Risk Neuroblastoma Susceptibility Loci. PloS Genet (2011) 7(3):e1002026. doi: 10.1371/journal.pgen.1002026

Conflict of Interest: The authors declare that the research was conducted in the absence of any commercial or financial relationships that could be construed as a potential conflict of interest.

Copyright (c) 2021 Chen, Guan, Guo, Miao and Zhuo. This is an open-access article distributed under the terms of the Creative Commons Attribution License (CC BY). The use, distribution or reproduction in other forums is permitted, provided the original author(s) and the copyright owner(s) are credited and that the original publication in this journal is cited, in accordance with accepted academic practice. No use, distribution or reproduction is permitted which does not comply with these terms. 